13

\title{
Влияние наночастиц серебра на фотодетектирующие свойства нанокомпозита $\mathrm{TiO}_{2}$ /оксид графена
}

\author{
() Е.В. Селиверстова, Н.Х. Ибраев, А.Ж. Жумабеков \\ Институт молекулярной нанофотоники, Карагандинский государственный университет им. Е.А. Букетова, \\ 100028 Караганда, Казахстан \\ e-mail: genia_sv@mail.ru, niazibrayev@mail.ru
}

Поступила в редакцию 21.04.2020 г.

В окончательной редакции 30.04.2020 г.

Принята к публикации 20.05.2020 г.

\begin{abstract}
Изучено влияние наночастиц серебра на оптоэлектронные и фотоэлектрические свойства нанокомпозитного материала на основе оксида графена $(\mathrm{O \Gamma})$ и $\mathrm{TiO}_{2}$. Электронной микроскопией и спектроскопией комбинационного рассеяния показано, что при гидротермальном синтезе происходит образование нанокомпозитного материала $\mathrm{TiO}_{2}-\mathrm{O}$. Спектр поглощения нанокомпозита сдвинут в длинноволновую область относительно поглощения $\mathrm{TiO}_{2}$. Вольт-амперные характеристики фотодетектора на основе пленок нанокомпозита $\mathrm{TiO}_{2}$-ОГ без наночастиц $\mathrm{Ag}$ и с добавлением таковых увеличиваются соответственно в 2 и 7.5 раз относительно чистого диоксида титана. Оптоэлектронные параметры устройств также увеличились, что связано с увеличением подвижности носителей заряда в нанокомпозитных пленках.
\end{abstract}

Ключевые слова: нанокомпозит, оксид графена, диоксид титана, фотодетектор, наночастицы Ag.

DOI: $10.21883 /$ OS.2020.09.49875.135-20

\section{Введение}

Оксиды переходных металлов открывают большие возможности в оптоэлектронных приложениях, где необходима высокая чувствительность к ультрафиолетовому излучению. Фотоприемники ультрафиолетового излучения могут быть использованы как для гражданских, так и военных целей, включая биологический и химический анализ, мониторинг окружающей среды $[1,2]$. Ультрафиолетовые фотоприемники обычно изготавливаются из широкозонных полупроводниковых материалов, таких как $\mathrm{TiO}_{2}$ [3-5], $\mathrm{ZnO}$ [6] и $\mathrm{ZnS}$ [7].

Диоксид титана $\mathrm{TiO}_{2}$ является одним из наиболее изученных полупроводников благодаря высокой перспективности его применения в фотокатализе, фотовольтаических элементах и газовых сенсорах [8-10]. Диоксид титана с шириной запрещенной зоны $3.2 \mathrm{eV}$ чувствителен к свету с длинами волн ниже $380 \mathrm{~nm}$. Это позволяет использовать его для изготовления фотодетекторов для УФ диапазона. Многими авторами показано, что при допировании $\mathrm{TiO}_{2}$ углеродными наноструктурами можно увеличить как чувствительность, так и производительность устройств на его основе. Для этой цели были изучены углеродные материалы различной природы, такие как активированный уголь, сажа, углеродные нити, нанотрубки [11,12].

Однако наиболее перспективным, на наш взгляд, является использование оксида графена (ОГ) и его производных [11-16]. В работе [17] нами было продемонстрировано, что добавление ОГ и восстановленного ОГ в $\mathrm{TiO}_{2}$ приводит к росту его фотокаталитической активности почти в 10 раз, что является результатом повышения адсорбционных характеристик, улучшения фотоэлектрических параметров и эффективности светосбора синтезированных нанокомпозитов.

В настоящее время в фотодетектирующих устройствах графен и диоксид титана обычно используются в виде слоистой структуры либо в комбинации с наноструктурами полупроводника (наностержни, наносферы, дорожки) [18-20]. Между тем работы по изучению фотодетекторов на основе нанокомпозитов $\mathrm{TiO}_{2}$ и ОГ, распределенного по объему полупроводника, практически отсутствуют. В настоящей работе представлены результаты исследования оптоэлектронных и фотоэлектрических показателей такого фотодетектора. Показано, что, используя широкодоступный и удобный в использовании ОГ, можно значительно повысить показатели УФ детектора на основе $\mathrm{TiO}_{2}$.

Внедрение плазмонных наночастиц серебра в нанокомпозитные слои позволяет улучшить фотодетектирующие свойства синтезированного материала. Считается, что включение металлических плазмонных наноструктур в графен усиливает взаимодействие свет-вещество [21,22]. Такие наноструктуры были использованы для поверхностно-усиленной спектроскопии комбинационного рассеяния (SERS), спектроскопии одиночных молекул, улучшения фотодетектирования, фотовольтаики и светоизлучающих устройств.

В работах [23-29] было показано, что присутствие плазмонных наночастиц $\mathrm{Ag}$ или $\mathrm{Au}$ усиливает как фотокаталитические, так и фотовольтаические характеристики нанокомпозитов $\mathrm{TiO}_{2}$-графен. При этом оптимизация фотореакций в присутствии плазмонов в полупроводниках приписывается росту генерации и разделения 
электрон-дырочных пар (ЭДП), а также расширению фотоактивности материала в направлении видимого диапазона света [26-29]. Мы также предприняли попытку использовать плазмонный эффект наночастиц серебра для того, чтобы увеличить оптоэлектронные параметры детекторов на основе нанокомпозита $\mathrm{TiO}_{2}$-ОГ.

\section{Экспериментальная часть}

Нанокомпозит на основе $\mathrm{TiO}_{2}$ и ОГ был синтезирован гидротермальным методом по методике работ $[17,30]$. Для приготовления был использован ОГ (SLGO, Cheaptubes) и $\mathrm{TiO}_{2}(d>21 \mathrm{~nm}$, анатаз, 99.7\%, Sigma Aldrich), деионизованная вода (система очистки воды AquaMax), этанол (безводный). Концентрация ОГ в нанокомпозите была равна $5 \mathrm{wt} \%$ по отношению к $\mathrm{TiO}_{2}$, поскольку ранее было показано, что при указанном соотношении регистрируется наибольшее увеличение зарядо-транспортных параметров и фотокаталической активности синтезированного нанокомпозита [17].

Морфология поверхности и микроструктура полученного композита была исследована на сканирующем электронном микроскопе (CЭM) Mira 3MLU (Tescan) и просвечивающем электронном микроскопе (ПЭМ) JEM$2100 \mathrm{~F}$ (Jeol) с ускоряющим напряжением $120 \mathrm{kV}$.

Наночастицы (НЧ) Ag в этиловом спирте получены абляцией мишени серебра второй гармоникой твердотельного Nd:YAG-лазера (SOLAR LQ215, $\lambda_{\text {gen }}=532 \mathrm{~nm}$, $\tau=7 \mathrm{~ns}, v=20 \mathrm{~Hz}$ ) по методике работы [31]. Согласно данным СЭМ, они имеют сферическую форму. Концентрация НЧ серебра в рабочем растворе составила $C_{\mathrm{Ag}}=1 \cdot 10^{-10} \mathrm{~mol} / \mathrm{L}$. Средний диаметр НЧ определен методом динамического рассеяния света с помощью анализатора Nanosizer 90S (Malvern) и равен $31 \pm 7 \mathrm{~nm}$.

Пленки из $\mathrm{TiO}_{2}$ или нанокомпозита $\mathrm{TiO}_{2}-\mathrm{O}$ были приготовлены из пасты, полученной при непрерывном перемешивании $150 \mathrm{mg}$ порошка $\mathrm{TiO}_{2}$ или $\mathrm{TiO}_{2}$-ОГ c $1 \mathrm{~mL}$ этанола в течение $24 \mathrm{~h}$. Нанокомпозитные пленки c плазмонными НЧ были получены при добавлении необходимого количества растворов НЧ Ag в подготовленную пасту и их дополнительном перемешивании в течение $24 \mathrm{~h}$. Концентрация НЧ $\mathrm{Ag}$ в таких пастах была равна $10^{-11}, 10^{-12}$ или $10^{-13} \mathrm{~mol} / \mathrm{L}$.

Готовую пасту наносили на поверхность подложек методом „spin-coating“ при скорости вращения $2000 \mathrm{rpm}$. Средняя толщина пленок, согласно СЭМ, равна $4.2 \mu \mathrm{m}$. После нанесения пленку отжигали в $\mathrm{Ar}$ атмосфере в течение $2 \mathrm{~h}$ при температуре $450^{\circ} \mathrm{C}$. Для проведения оптических измерений пленки были сформированы на кварцевых подложках.

Для сборки фотодетектора были использованы стекла с проводящим слоем фторированного оксида олова (FTO, $7 \Omega$ sq, Sigma Aldrich). На поверхности подложек были вырезаны дорожки встречно-гребенчатого типа (рис. 1) с помощью лазерного скрайбера BLS0503MM (Bodor). Расстояние между дорожками равно $1.5 \mathrm{~mm}$, длина дорожек - $10 \mathrm{~mm}$. СЭМ-измерения показали, что при скрайбировании формируются дорожки шириной 250-280 $\mu \mathrm{m}$, при нанесении пасты пленка формируется поверх канала.

Для регистрации спектров комбинационного рассеяния (КР) нанокомпозитного материала был использован микроскоп Confotec MR520 (Sol Instruments) с лазерным возбуждением на длине волны $632.8 \mathrm{~nm}$.

Измерения вольт-амперных характеристик (BAX) приготовленных образцов проводили с помощью потенциостата-гальваностата Elins P-20X (Elins) при облучении образцов светом ксеноновой лампы с мощностью $35 \mathrm{~mW} / \mathrm{cm}^{2}$.

Для исследования времени отклика образцов использовалась установка, схема которой показана на рис. 2, и импульсный лазер LQ215 (SOLAR) с генератором третьей гармоники с $\lambda_{\text {gen }}=355 \mathrm{~nm}$ и длительностью импульса $7 \mathrm{~ns}$.

При облучении детектора сигнал фотонапряжения образца подавался на цифровой осциллограф Bordo-211, максимальная чувствительность которого $10 \mathrm{mV} / \mathrm{div}$. При этом к образцу прикладывалось постоянное напряжение, равное 9V. Измерения проводились в режиме возбуждения одиночными импульсами. Для синхронизации запуска развертки осциллографа с запуском возбуждающего импульса света и обеспечения заданного времени задержки использовалось программное и аппаратное обеспечение на базе Arduino. Значение постоянной $R C$-цепи измерительной схемы не превышает $1 \mu \mathrm{s}$, а экспериментально подобранное время задержки составляло $100 \mu \mathrm{s}$.

\section{Результаты и их обсуждение}

При исследовании микроструктуры нанокомпозитного материала и его исходных компонентов были получены данные, показанные на рис. 3. Из рисунка видно, что в образце диоксида титана наночастицы агрегированы. На снимке можно различить межчастичные поры. Оксид графена представлен в виде слоистых хлопьев, где листы склонны к формированию „морщин“ и складок из-за кислородсодержащих групп в его структуре. В нанокомпозите хорошо различимы тонкие листы ОГ, распределенные на поверхности наночастиц $\mathrm{TiO}_{2}$, а также между ними, что видно из ПЭМ. При этом видно, что после гидротермального синтеза частицы $\mathrm{TiO}_{2}$ располагаются не просто на поверхности ОГ, они ими „окутаны“. Это позволит частицам $\mathrm{TiO}_{2}$ под действием света инжектировать свои фотогенерированные электроны в листы ОГ, обеспечивая их эффективный транспорт к слою FTO и дальнейшую регистрацию [30].

Наночастицы серебра практически неразличимы на изображениях пленок нанокомпозита в силу их малой концентрации, поэтому их наличие было подтверждено данными энерго-дисперсионного анализа (ЭДС, рис. $3, e)$. 

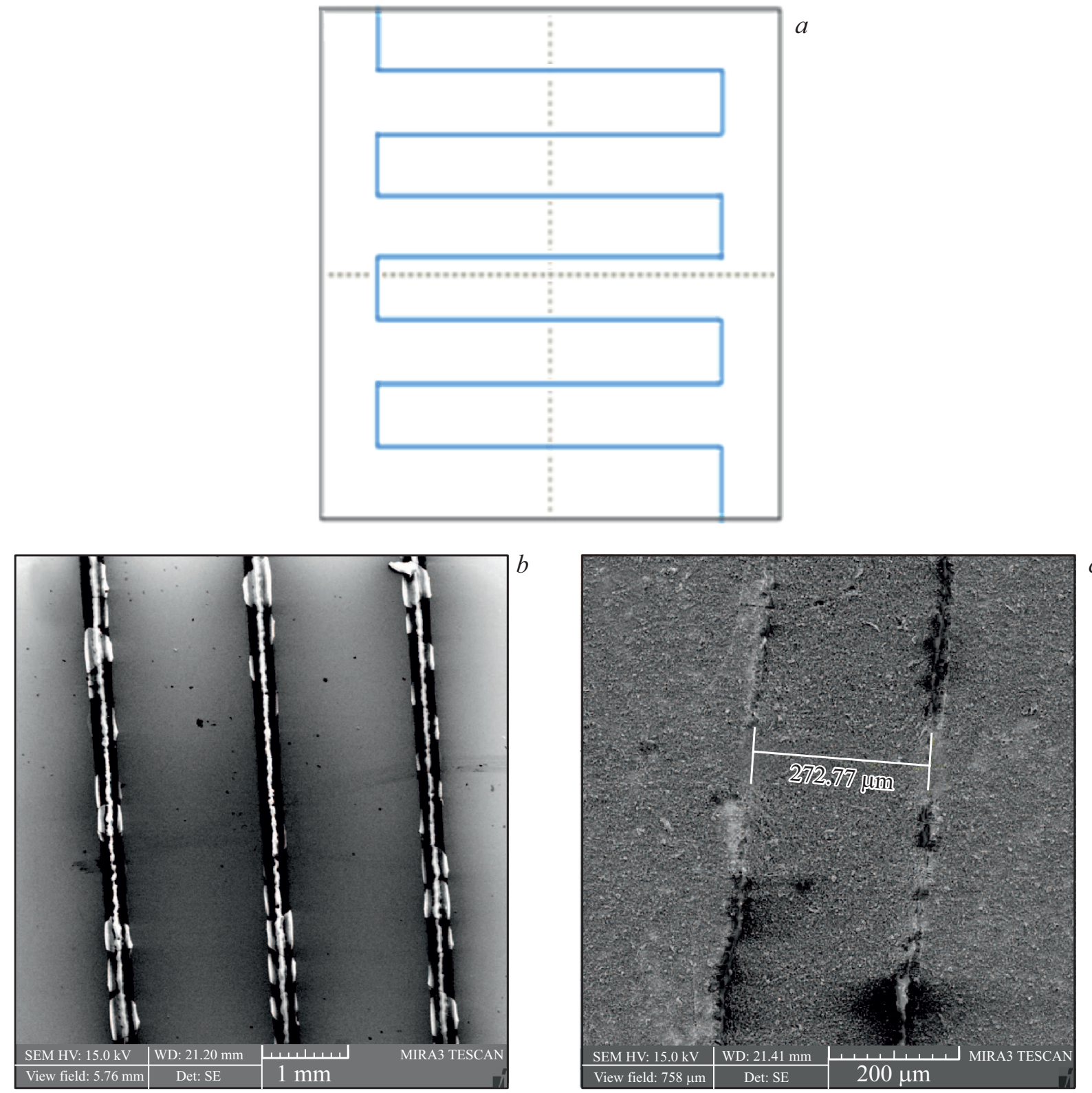

Pис. 1. Схема дорожек $(a)$ и СЭМ-изображения $(b, c)$ дорожек на поверхности $\mathrm{FTO}$ и пленки $\mathrm{TiO}_{2}$ на поверхности дорожки $(c)$.

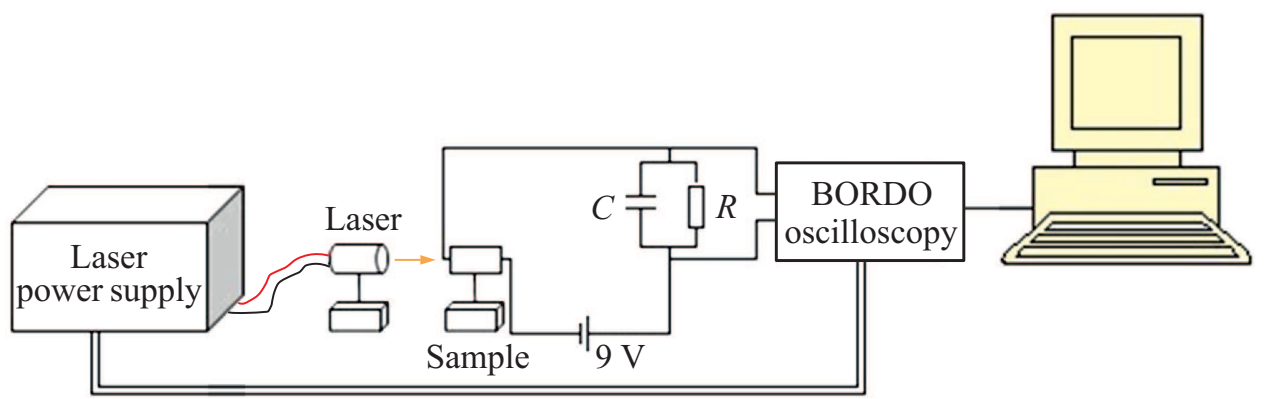

Рис. 2. Блок-схема экспериментальной установки для исследования времени отклика. 

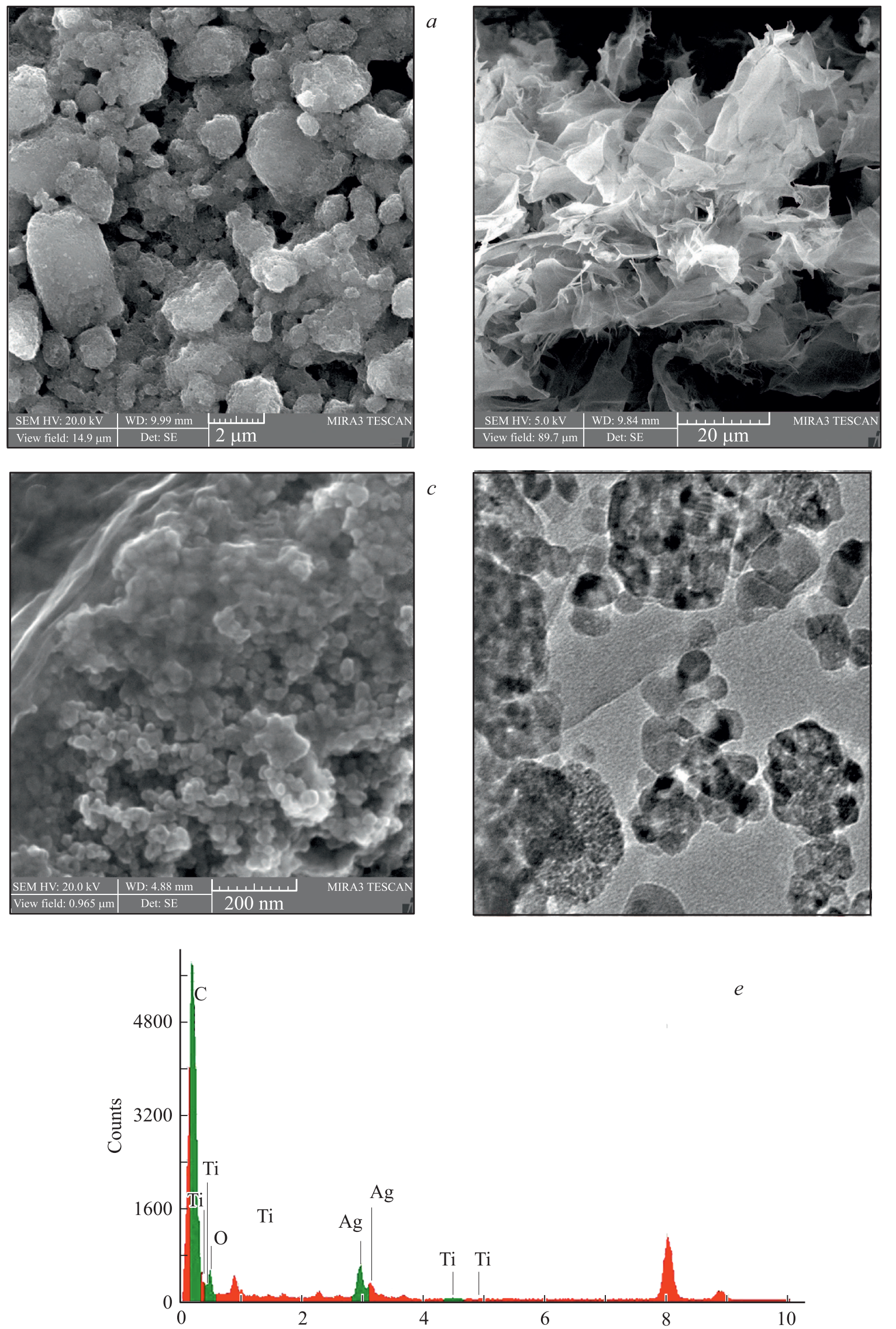

Рис. 3. СЭМ- $(a-c)$ и ПЭМ-изображение $(d)$ нанокомпозита $\mathrm{TiO}_{2}(a)$, ОГ $(b), \mathrm{TiO}_{2}-\mathrm{O}$ (c, $\left.d\right)$ и ЭДС-анализ $(e) \mathrm{TiO}_{2}-\mathrm{OГ} \mathrm{c} \mathrm{HЧ} \mathrm{Ag}$. 

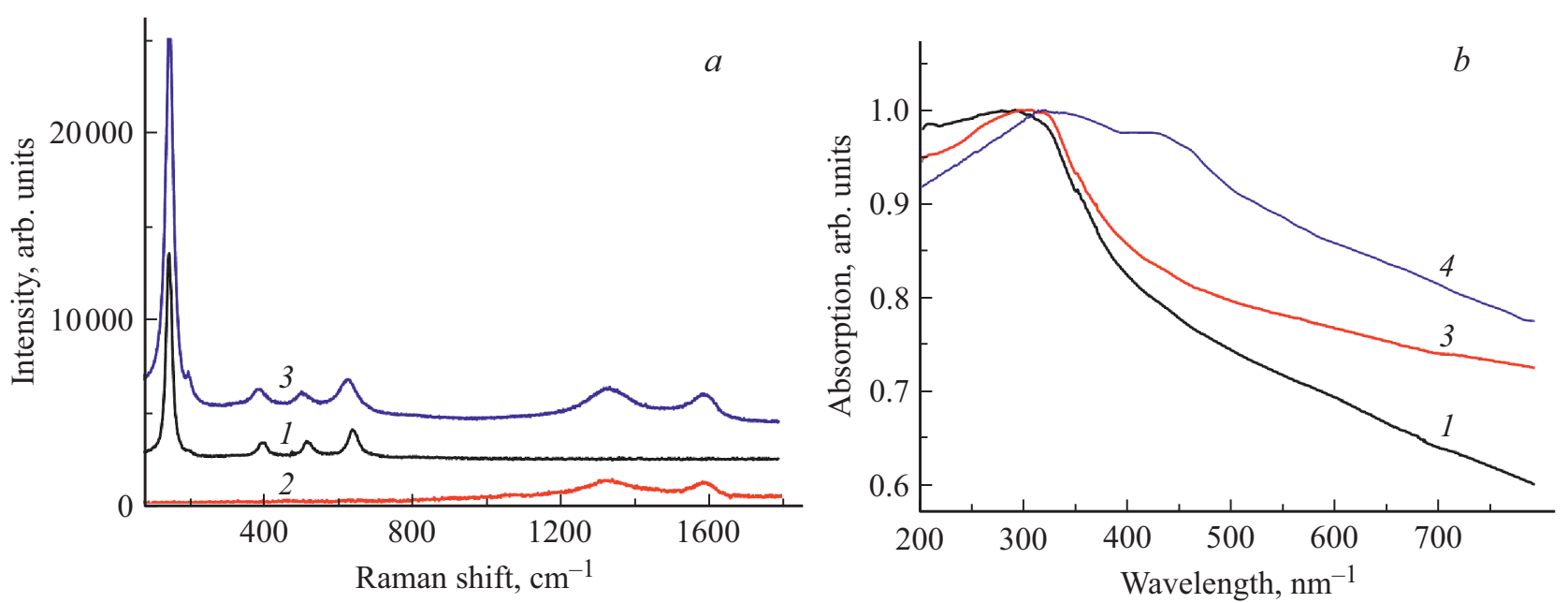

Рис. 4. КР-спектры $(a)$ и спектры поглощения пленок $(b): 1-\mathrm{TiO}_{2}, 2-\mathrm{OГ}, 3-\mathrm{TiO}_{2}-\mathrm{O}$, $4-\mathrm{TiO}$-ОГ $+\mathrm{Ag}$ при $C_{\mathrm{Ag}}=10^{-11} \mathrm{~mol} / \mathrm{L}$.

Также нами были зарегистрированы спектры КР исследуемых образцов (рис. 4). Диоксид титана анатазной структуры имеет шесть комбинационно-активных пиков в колебательном спектре [30,32]. Среди них - три пика $E_{g}$ около 150,180 и $630 \mathrm{~cm}^{-1}$, два пика $B_{1 g}$ при 395 и $505 \mathrm{~cm}^{-1}$, а также пик $A_{1 g}$ на $481 \mathrm{~cm}^{-1}$. В спектре ОГ присутствуют две характерные полосы: $D$-полоса около $1350 \mathrm{~cm}^{-1}$, которая характеризует степень дефектности графена, и $G$-полоса около $1590 \mathrm{~cm}^{-1}$. Отношение интенсивностей $I_{\mathrm{D}} / I_{\mathrm{G}}$ равно 1.2 . КР-спектры нанокомпозита представляют собой комбинацию спектров отдельных составляющих. Отношение интенсивностей $I_{\mathrm{D}} / I_{\mathrm{G}}$ в нанокомпозитном материале равно 1.03. Уменьшение данного параметра указывает на процесс восстановления ОГ в ходе синтеза, где может происходить образование углеродных $s p^{2}$-доменов и уменьшение числа кислородсодержащих групп [33].

Измерения оптических свойств исследуемых пленок показали, что в присутствии ОГ наблюдается батохромный сдвиг края полосы поглощения $\mathrm{TiO}_{2}$. В спектре поглощения нанокомпозита с $\mathrm{HЧ} \mathrm{Ag}$ регистрируется очень слабый максимум около $420 \mathrm{~nm}$. При этом в спектрах исходных компонентов таких изменений нет. В частности, край полосы поглощения диоксида титана приходится на $380 \mathrm{~nm}$, а в спектре поглощения ОГ различим максимум около $230 \mathrm{~nm}[34,35]$. Известно, что полоса поглощения на $230 \mathrm{~nm}$ сформирована переходами между орбиталями $\pi \pi^{*}$-природы в ароматических связях $\mathrm{C}-\mathrm{C}$. Длинноволновый сдвиг края полосы поглощения нанокомпозита связан с изменением ширины запрещенной зоны такого материала по сравнению с чистым $\mathrm{TiO}_{2}$ [30]. Это может привести к более высокой светопоглощающей способности нанокомпозита $\mathrm{TiO}_{2}$-ОГ.

Вольт-амперные характеристики измеренных образцов, а также некоторые из их оптоэлектронных параметров показаны на рис. 5, $a$ и в табл. 1. Вольтамперные характеристики были измерены как при по-
Таблица 1. Оптоэлектронные параметры пленок на основе $\mathrm{TiO}_{2}, \mathrm{TiO}_{2}$-ОГ и нанокомпозитов с добавлением НЧ Ag

\begin{tabular}{l|c|r|r}
\hline \multicolumn{1}{c|}{ Образец } & $\begin{array}{c}I_{\mathrm{ph}}, \mu \mathrm{A} \\
\text { при }+30 \mathrm{~V}\end{array}$ & \multicolumn{1}{c|}{$R, \mathrm{~A} / \mathrm{W}$} & $D^{*}$, Jones \\
\hline $\mathrm{TiO}_{2}$ & 1.10 & $31.0 \cdot 10^{-6}$ & $6.3 \cdot 10^{7}$ \\
$\mathrm{TiO}_{2}$-ОГ & 1.98 & $56.0 \cdot 10^{-6}$ & $6.2 \cdot 10^{7}$ \\
$\mathrm{TiO}_{2}-\mathrm{O}+\mathrm{Ag}\left(10^{-13}\right)$ & 8.30 & $237.0 \cdot 10^{-6}$ & $12.0 \cdot 10^{7}$ \\
$\mathrm{TiO}_{2}-\mathrm{O}+\mathrm{Ag}\left(10^{-12}\right)$ & 0.78 & $22.3 \cdot 10^{-6}$ & $6.4 \cdot 10^{7}$ \\
$\mathrm{TiO}_{2}$-ОГ $+\mathrm{Ag}\left(10^{-11}\right)$ & 0.40 & $11.4 \cdot 10^{-6}$ & $0.1 \cdot 10^{7}$
\end{tabular}

ложительном смещении напряжения (до $+30 \mathrm{~V}$ ), так и при отрицательных значениях - до $-5 \mathrm{~V}$. Кривые зависимости $I(U)$ имеют нелинейную форму. При этом даже в отсутствие освещения образцов регистрируются большие значения тока.

При фотооблучении образцов $\mathrm{TiO}_{2}$ было зарегистрировано максимальное значение тока, равное $2 \mu \mathrm{A}$. Значения генерируемого фототока $I_{\mathrm{ph}}$ детектора, вычисленные по разнице темновых и световых значений $I$, показаны в табл. 1 . Из рисунка видно, что значения $I_{\mathrm{ph}}$ для пленок на основе $\mathrm{TiO}_{2}$-ОГ почти в 3 раза выше, однако из-за большого значения темнового тока величина $I_{\mathrm{ph}}$ такого фотодетектора по сравнению с чистым $\mathrm{TiO}_{2}$ увеличивается лишь в 1.8 раза. Для фотодетекторов с плазмонными НЧ значения фототока возросли только при концентрации $\mathrm{Ag}$, равной $10^{-13} \mathrm{~mol} / \mathrm{L}$. При этом видно, что и темновые, и световые токи увеличились почти пропорционально. В дальнейшем при упоминании параметров фотодетектора $\mathrm{TiO}_{2}-\mathrm{O} \Gamma+\mathrm{Ag}$ будет подразумеваться именно эта концентрация НЧ серебра.

Усиление фототока детектора $I_{\mathrm{ph}}$ на основе трехкомпонентной пленки составило 4.2 раза относительно пленок $\mathrm{TiO}_{2}$-ОГ и 7.5 раз относительно чистого диоксида титана. При дальнейшем добавлении $\mathrm{HЧ} \mathrm{Ag}$ в 


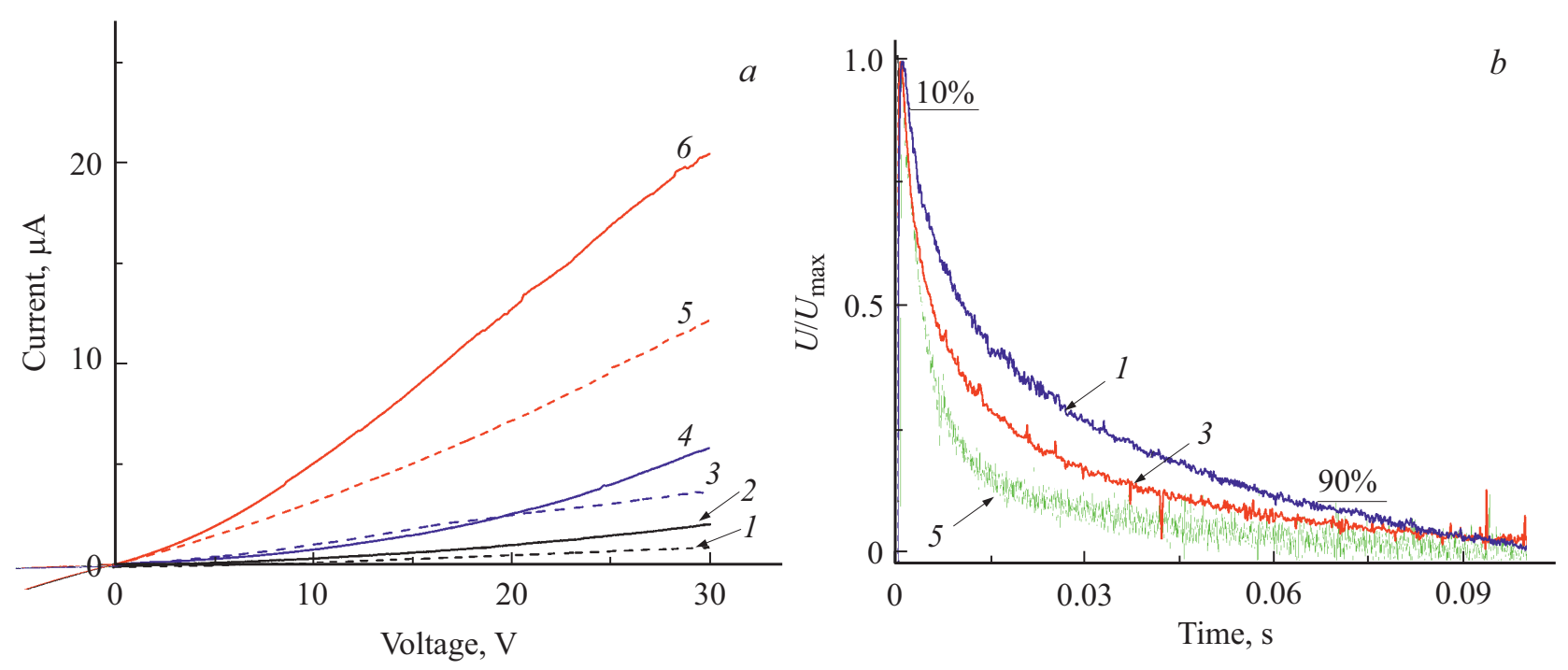

Рис. 5. Темновые $(1,3,5)$ и световые $(2,4,6)$ вольт-амперные характеристики $(a)$ и кинетики генерации и затухания носителей заряда $(b)$ пленок на основе: $1,2-\mathrm{TiO}_{2}, 3,4-\mathrm{TiO}_{2}-\mathrm{O}, 5,6-\mathrm{TiO}_{2}-\mathrm{O} \Gamma+\mathrm{Ag}$ при $C_{\mathrm{Ag}}=10^{-13} \mathrm{~mol} / \mathrm{L}$.

Таблица 2. Временны́е характеристики пленок на основе $\mathrm{TiO}_{2}, \mathrm{TiO}_{2}$-ОГ и нанокомпозитов с добавлением $\mathrm{HЧ} \mathrm{Ag}$

\begin{tabular}{l|r|l|l}
\hline \multicolumn{1}{c|}{ Образец } & \multicolumn{1}{c|}{$t_{\text {grow }}, \mathrm{s}$} & \multicolumn{1}{c}{$t_{1}, \mathrm{~s}$} & \multicolumn{1}{c}{$t_{2}, \mathrm{~s}$} \\
\hline $\mathrm{TiO}_{2}$ & $40.5 \cdot 10^{-3}$ & 0.95 & 1.9 \\
$\mathrm{TiO}_{2}-\mathrm{OГ}$ & $27.7 \cdot 10^{-3}$ & 0.45 & 1.84 \\
$\mathrm{TiO}_{2}-\mathrm{O}-\mathrm{Ag}\left(10^{-13}\right)$ & $0.7 \cdot 10^{-3}$ & $0.25 \cdot 10^{-3}$ & $2.4 \cdot 10^{-3}$ \\
$\mathrm{TiO}_{2}-\mathrm{O}-\mathrm{Ag}\left(10^{-12}\right)$ & $3.2 \cdot 10^{-6}$ & $0.14 \cdot 10^{-3}$ & $1.5 \cdot 10^{-3}$ \\
$\mathrm{TiO}_{2}-\mathrm{O}-\mathrm{Ag}\left(10^{-11}\right)$ & $49.0 \cdot 10^{-6}$ & $0.85 \cdot 10^{-3}$ & $3.7 \cdot 10^{-3}$
\end{tabular}

пленку нанокомпозита регистрируется уменьшение $I_{\mathrm{ph}}$, что связано с малыми значениями как темнового, так и светового тока. В частности, значения $I_{\text {dark }}$ составили $12.14,0.38$ и $0.78 \mu \mathrm{A}$ соответственно для концентраций серебра от $10^{-13}$ до $10^{-11} \mathrm{~mol} / \mathrm{L}$. Как видно, линейной зависимости $I_{\text {dark }}$ от концентрации НЧ Ag нет. Однако известно, что плотность дрейфового тока прямо пропорциональна удельной проводимости полупроводника. Поэтому можно сделать вывод, что при малых концентрациях НЧ $\mathrm{Ag}$ этот показатель увеличивается. В то же время проводимость зависит и от дрейфовой скорости или подвижности носителей заряда.

На основании полученных данных пока сложно сделать вывод, какой механизм является доминирующим в увеличении фототока детектора на основе нанокомпозита с добавлением плазмонных НЧ, в связи с чем далее была изучена кинетика генерации и транспорта носителей заряда в приготовленных фотодетекторах (рис. 5,b). Из кинетических данных видно, что время нарастания фотонапряжения во всех пленках значительно меньше времени затухания. Полная кинетика затухания напряжения в исследуемых пленках может быть описана с помощью биэкспоненциального уравнения.
Из кинетических данных были определены характерные времена (табл. 2): время нарастания $t_{\text {grow }}$ и времена жизни носителей заряда $t_{1}$ и $t_{2}$.

Из рисунка видно, что времена релаксации фотопроводимости в детекторе на основе $\mathrm{TiO}_{2}$ больше, чем для нанокомпозитных образцов. Для фотодетекторов с ОГ было зарегистрировано уменьшение времени нарастания кинетики напряжения по сравнению с чистым $\mathrm{TiO}_{2}$ на 46\%, времена жизни носителей заряда также уменьшились. Можно сделать вывод, что в образцах $\mathrm{TiO}_{2}-\mathrm{OГ}$ генерация и разделение носителей заряда происходит за меньшие времена. При этом в присутствии НЧ Ag происходит дальнейшее уменьшение значений как $t_{\text {grow }}$, так и $t_{1}$ и $t_{2}$.

Далее было определено время пролета $t_{\mathrm{tr}}$, которое соответствовало моменту выхода из образца центра тяжести дрейфующего пакета носителей заряда, а также определена их подвижность по методике, предложенной в работе [36]. Для этого строился двойной логарифмический график временной зависимости напряжения фотогенерации. Точка перегиба кривой принималась за время выхода из образца центра тяжести дрейфующего пакета носителей заряда $t_{\mathrm{tr}}$. Для образцов $\mathrm{TiO}_{2}$ это время равно $5.9 \mu$ и и 5.1 и $2.5 \mu \mathrm{s}$ для $\mathrm{TiO}_{2}-\mathrm{OГ}$ и $\mathrm{TiO}_{2}-\mathrm{O}+\mathrm{Ag}$ соответственно. Подвижность носителей заряда $\mu_{d}$ была оценена из выражения

$$
\mu_{d}=\frac{d}{t_{\mathrm{tr}} E}
$$

где $d-$ толщина пленки, $E-$ прикладываемое напряжение к образцу (9V).

Для пленки диоксида титана значение $\mu_{d}$ равно $7.9 \cdot 10^{-2} \mathrm{~m} / \mathrm{V} \cdot \mathrm{s}$. Тогда как для пленки на основе $\mathrm{TiO}_{2}$ ОГ это значение увеличилось до $9.1 \cdot 10^{-2} \mathrm{~m} / \mathrm{V} \cdot \mathrm{s}$ или в 1.15 раза, а при добавлении НЧ $\mathrm{Ag}$ - в 2.35 раза 
или до $18.6 \cdot 10^{-2} \mathrm{~m} / \mathrm{V} \cdot \mathrm{s}$. Таким образом, видно, что подвижность носителей заряда в фотодетекторах с ОГ увеличилась. Дальнейший рост $\mu_{d}$ наблюдается и при добавлении НЧ $\mathrm{Ag}$ в полупроводниковые пленки.

При оценке оптоэлектронных параметров приготовленных образцов была выполнена оценка чувствительности $R$, удельная детектирующая способность $D^{*}$ и время фотоотклика детекторов. Чувствительность $R$ фотодетекторов была вычислена с помощью формулы $R=I_{\mathrm{ph}} / P$, где $P-$ мощность падающего излучения. Из табл. 1 видно, что чувствительность композитных пленок в 1.8 раза выше, чем у пленок чистого $\mathrm{TiO}_{2}$. Наилучшая чувствительность фотодетектора наблюдается для пленок с добавлением $\mathrm{HЧ} \mathrm{Ag}$ той же концентрации, что и для значений фототока. При этом чувствительность такой пленки составляет $\sim 0.24 \mathrm{~mA} / \mathrm{W}$, что в 4.2 раза выше, чем у пленок без добавления НЧ.

Далее была оценена удельная детектирующая способность приготовленных образцов $D^{*}$, которая определяет способность устройства детектировать слабые световые сигналы и может быть определена $[37,38]$ из выражения

$$
D^{*}=\frac{R A^{1 / 2}}{\sqrt{2 e I_{\text {dark }}}},
$$

где $R$ - чувствительность пленок, $A$ - освещаемая площадь образца, $e-$ заряд электрона по модулю, $I_{\text {dark }}-$ значение темнового тока. Полученные значения как для чистого $\mathrm{TiO}_{2}$, так и для $\mathrm{TiO}_{2}$-ОГ равны $\sim 6 \cdot 10^{7}$ Jones. При минимальном содержании НЧ $\mathrm{Ag}$ в пленке детектирующая способность фотодетектора увеличилась почти в 2 раза. Этот факт указывает на то, что увеличение данного параметра связано со значительным ростом генерируемого фототока, несмотря на значительную величину $I_{\text {dark}}$, как это наблюдается в системе без плазмонов.

Сравнивая полученные значения фототока, $R$ и $D^{*} \mathrm{c}$ данными других авторов [39,40], можно отметить, что пленки $\mathrm{TiO}_{2}$-ОГ не уступают по показателям образцам на основе чистого графена, и даже превышают значения генерируемого $I_{\mathrm{ph}}$.

Время фотоотклика детекторов (или время включения) было определено из кинетических данных как временной промежуток, в течение которого фотонапряжение или ток изменяются от 10 до $90 \%$ от своего максимального значения (рис. $5, b$ ), по методике работ $[37,40]$. Для образца $\mathrm{TiO}_{2}$ этот параметр равен $58 \mu \mathrm{s}$. Для пленок с ОГ и ОГ $+\mathrm{Ag}$ время фотоотклика уменьшается до 39 и $9.5 \mu \mathrm{s}$ соответственно. Таким образом, время включения полупроводникового детектора на основе $\mathrm{TiO}_{2}$ может быть сокращено при добавлении ОГ или ОГ и плазмонных НЧ в $\sim 1.5$ и 6.1 раза соответственно. Стоит отметить, что полученные в настоящей работе значения времени фотоотклика меньше, чем для $\mathrm{TiO}_{2}-$ детектора с электродами $\mathrm{Ni}(11.43 \mathrm{~s})$ [41], а также для детекторов на основе слоистых структур $\mathrm{TiO}_{2}$-графен $(1.1 \mathrm{~s})$ [37] и графен-восстановленный ОГ $(114 \mu \mathrm{s})$ [42].
Коэффициент выпрямления фотодетектора, определенный из темновых характеристик по отношению $I_{1 \mathrm{~V}} / I_{-1 \mathrm{~V}}[40]$, был равен $0.92,3.03$ и 0.85 для пленок на основе чистого $\mathrm{TiO}_{2}, \mathrm{TiO}_{2}$-ОГ и $\mathrm{TiO}_{2}-\mathrm{O} \Gamma+\mathrm{Ag}$ соответственно. Видно, что исследуемые детекторы обладают слабыми выпрямляющими свойствами.

Как указано в работах $[17,38]$, улучшение фотоэлектрических параметров пленок $\mathrm{TiO} 2-\mathrm{OГ}$ является результатом улучшения зарядо-транспортных характеристик синтезированных пленок, а также ускорения процесса разделения зарядов в пленке. При этом листы графена служат транспортными „каналами“ для передачи носителей заряда к электроду съема. Действительно, при оценке сопротивления фотодетектора, вычисленного по закону Ома с использованием значений падения напряжения из кинетических данных, было обнаружено, что для детектора на основе $\mathrm{TiO}_{2}$ сопротивление равно $154.5 \mathrm{Ohm}$, тогда как для пленок $\mathrm{TiO}_{2}$-ОГ эта величина равна $32 \mathrm{Ohm}$. С добавлением $\mathrm{HЧ} \mathrm{Ag}$ сопротивление образцов уменьшилось до $20 \mathrm{Ohm}$.

Таким образом видно, что добавление ОГ в $\mathrm{TiO}_{2}$ позволяет понизить значения сопротивления пленки полупроводника в несколько раз, что согласуется с данными авторов работ [17,37], где было показано, что это достигается за счет перемещения электронов через листы ОГ. В присутствии НЧ Ag наблюдается дополнительное уменьшение сопротивления нанокомпозита транспорту электронов. Увеличение фотопроводимости пленок диоксида титана в присутствии ОГ также подтверждается ростом подвижности носителей заряда в нанокомпозитах как с НЧ $\mathrm{Ag}$, так и без таковых.

\section{Заключение}

Исследования показали, что при использовании нанокомпозитного материала на основе $\mathrm{TiO}_{2}$ и ОГ возможно улучшение токовых и оптоэлектронных параметров фотодетектора. При добавлении НЧ Ag эти параметры могут быть улучшены.

При исследовании вольт-амперных характеристик фотодетектора было показано, что в присутствии ОГ фототок устройства увеличивается в 1.8 раза. Для фотодетекторов с плазмонными НЧ значения фототока возросли только при концентрации $\mathrm{Ag}$, равной $10^{-13} \mathrm{~mol} / \mathrm{L}$. При этом увеличение фототока для трехкомпонентной пленки составило 4.2 раза относительно пленок $\mathrm{TiO}_{2}$-ОГ и 7.5 раз относительно чистого диоксида титана. Чувствительность $R$ и удельная детектирующая способность фотодетекторов с плазмонными НЧ также увеличиваются.

Показано, что рост фототока детектора связан преимущественно с ростом подвижности носителей заряда, как это следует из данных кинетики фотопроводимости. Сравнивая подвижности носителей заряда в $\mathrm{TiO}_{2}\left(\sim 10^{-5} \mathrm{~cm}^{2} / \mathrm{V} \cdot \mathrm{s} \quad[43]\right)$ и графене 
$\left(2.5 \cdot 10^{5} \mathrm{~cm}^{2} / \mathrm{V} \cdot \mathrm{s}[44]\right)$, можно отметить, что зарядотранспортные свойства нанокомпозита улучшились благодаря высокой подвижности заряда в графеновой компоненте.

Кроме того, в синтезированных пленках при добавлении как ОГ, так и НЧ Ag происходит уменьшение сопротивления транспорту электронов, что, конечно, говорит о росте зарядо-транспортных показателей синтезированных пленок. Это согласуется с ранее полученными нами результатами для нанокомпозита на основе $\mathrm{TiO}_{2}$ и восстановленного ОГ [30].

Усиление фототока в гибридных устройствах на основе графена и наночастиц серебра можно объяснить как усилением электрического поля вблизи НЧ $\mathrm{Ag}[45,46]$, так и рассеянием света НЧ серебра. Это поле может увеличивать поглощение нанокомпозита в видимой области спектра [46], а также приводить к повышению подвижности носителей заряда внутри графеновой компоненты нанокомпозита [47].

\section{Финансирование работы}

Данная работа выполнена в рамках научно-исследовательского гранта АР05132443, финансируемых Министерством образования и науки Республики Казахстан.

\section{Благодарности}

Авторы благодарят Д.А. Афанасьева за консультации при проведении импульсных измерений и обсуждении полученных результатов.

\section{Конфликт интересов}

Авторы заявляют, что у них нет конфликта интересов

\section{Список литературы}

[1] Yu X., Zhao Z., Zhang J., Guo W., Qiu J., Li D., Li Z., Mou X., Li L., Li A., Liu H. // Small. 2011. V. 12. N 20. P. 2759. doi 10.1002/smll.201503388

[2] Peng M., Liu Y., Yu A., Zhang Y., Liu C., Liu J., Wu W., Zhang K., Shi X., Kou J., Zhai J., Wang Z.L. // ACS Nano. 2016. V. 10. N 1. P. 1572. doi 10.1021/acsnano.5b07217

[3] Wang Q., Li J.J., Gu C.Z. // J. Phys. Chem. C. 2012. V. 116. N 32. P. 16864. doi 10.1021/jp304193z

[4] Han Y., Wu G., Li H., Wang M., Chen H. // Nanotechn. 2010. V. 21. N 18. P. 185708. doi 10.1088/0957-4484/21/18/185708

[5] Wang L.B., Yang W.Y., Chong H.N., Wang L., Gao F.M., Tian L.H., Yang Z.B. // RSC Adv. 2015. V. 5. N 65. P. 52388. doi 10.1039/C5RA05861A

[6] Xue H.L., Kong X.Z., Liu Z.R., Liu C.X., Zhou J.R., Chen W.Y., Ruan S.P., Xu Q.T. // Appl. Phys. Lett. 2007. V. 90. N 20. P. 201118. doi 10.1063/1.2741128

[7] Liang Y., Liang H., Xiao X.D., Hark S.K. // J. Mater. Chem. 2012. V. 22. N 3. P. 1199. doi $10.1039 / \mathrm{c} 1 \mathrm{jm} 13903 \mathrm{~g}$

[8] Jang H.D., Kim S.K., Chang H., Jo E.H., Roh K.M., Choi J.H., Choi J.-W. // Aerosol Sci. Techn. 2015. V. 49. N 7. P. 538. doi 10.1080/02786826.2015.1050086
[9] Molina-Mendoza A., Moya A., Frisenda R., Svatek S.A., Gant P., Gonzalez-Abad S., Antolin E., Agrait N., Bollinger G.R., Perez de Lara D., Vilatela J., CastellanosGomez A. // J. Mat. Chem. C. 2016. V. 4. P. 10707. doi 10.1039/C6TC02344Da

[10] Ibrayev N., Seliverstova E., Omarova G. // Mat. Today: Proceed. 2020. V. 25. N 1. P. 39. doi 10.1016/j.matpr.2019.11.016

[11] Leary R., Westwood A. // Carbon. 2011. V. 49. N 3. P.741. doi 10.1016/j.carbon.2010.10.010

[12] Takeda N., Iwata N., Torimoto T., Yoneyama H. // J. Catal. 1998. V. 177. P. 240. doi 10.1006/jcat.1998.2117

[13] Ng Y.H., Lightcap I.V., Goodwin K., Matsumura M., Kamat P.V. // J. Phys. Chem. Lett. 2010. V. 1. P. 2222. doi $10.1021 / \mathrm{jz} 100728 \mathrm{z}$

[14] Li Q., Guo B.D., Yu J.G., Ran J.R., Zhang B.H., Yan H.J. // J. Am. Chem. Soc. 2011. V. 133. N 28. P. 10878. doi $10.1021 /$ ja2025454

[15] Zhang H., Lv X.J., Li Y.M., Wang Y., Li J.H. // ACS Nano. 2010. V. 4. N 1. P. 380. doi $10.1021 / \mathrm{nn} 901221 \mathrm{k}$

[16] Zhou Y., Bao Q.L., Tang L.A.L., Zhang Y.L., Loh K.P. // Chem. Mater. 2009. V. 21. N 13. P. 2950. doi $10.1021 / \mathrm{cm} 9006603$

[17] Ibrayev N., Zhumabekov A., Ghyngazov S., Lysenko E. // Mat. Res. Expr. 2019. V. 6. N 12. P. 125036. doi 10.1088/2053-1591/ab51a3

[18] Zhang D., Jing F., Gao F., Shen L., Sun D., Zhou J., Chen Y., Ruan S. // RSC Adv. 2015. V. 5. P. 83795(1). doi 10.1039/C5RA17023K.

[19] Yu X., Li Y., Hu X., Zhang D., Tao Y., Liu Z., He Y., Azimul Haque Md., Liu Zh., Wu T., Wang Q.L. // Nat. Commun. 2018. V. 9. P. 4299. doi 10.1038/s41467-018-06776-Z

[20] Wang M.Zh., Liang F.X., Nie B., Zeng L.H., Xia Zheng L., P. Lv, Yong Qiang Yu, Ch. Xie, Ya. Yang Li, Lu L.B. // Part. Part. Syst. Charact. 2013. V. 30. N 7. P. 630. doi $10.1002 /$ ppsc. 201300040

[21] Ozbay E. // Science. 2006. V. 311. P. 189. doi 10.1126/science.1114849

[22] Liu W.L., Lin F.C., Yang Y.C., Huang C.H., Gwo S., Huang M.H., Huang J. // Nanoscale. 2013. V. 5. P. 7953. doi 10.1039/C3NR02800C

[23] Wang T., Tang T., Gao Y., Chen Q., Zhang Zh., Bian H. // Physica E. 2019. V. 112. P. 128. doi 10.1016/j.physe.2018.10.033

[24] Fu N., Ren X., Wan J.-X. // J. Nanomater. 2019. N 8175803. P. 1. doi $10.1155 / 2019 / 8175803$

[25] Vasilaki E., Georgaki I., Vernardou D., Vamvakaki M., Katsarakis N. // J. Appl. Surf. Sci. 2015. V. 353. N 30. P. 865. doi 10.1016/j.apsusc.2015.07.056

[26] Lee J.-H., Kim I.-K., Cho D., Y. J.-I., K. Y.-J., Oh H.-J. // Carbon Lett. 2015. V. 16. N 4. P. 247. doi 10.5714/CL.2015.16.4.247

[27] Tian H., Wan Ch., Xue X., Hu X., Wang X. // Catalysts. 2017. V. 7. N 156. P. 1. doi 10.3390/catal7050156

[28] Gao W., Wang M., Ran Ch., Yao X., Yang H., Liu J., He D., Bai J. // Nanoscale. 2014. V. 6. P. 5498. doi $10.1039 / \mathrm{C} 3 \mathrm{NR} 05466 \mathrm{G}$

[29] Afanasyev D.A., Ibrayev N.Kh., Serikov T.M., Zeinidenov A.K. // Rus. J. Phys. Chem. A. 2016. V. 90. N 6. P. 833. doi 10.1134/S0036024416040026 
[30] Zhumabekov A.Zh., Ibrayev N.Kh., Seliverstova E.V. // Theor. Exp. Chem. 2020. V. 55. N 6. P. 398. doi 10.1007/s11237020-09632-8

[31] Ibrayev N., Ishchenko A., Afanasyev D., Zhumabay N. // Appl. Phys. B. 2019. V. 125. N 182. P. 1. doi 10.1007/s00340019-7292-y

[32] Swamy V., Kuznetsov A., Dubrovinsky L.S., Caruso R.A., Shchukin D.G., Muddle B.C. // Phys. Rev. B. 2005. V. 71. P. 184302. doi 10.1103/PhysRevB.71.184302

[33] Jorio A., Saito R., Dresselhaus G., Dresselhaus M.S. Raman Spectroscopy in Graphene Related Systems. Berlin: WileyVCH, 2011. $368 \mathrm{p}$.

[34] Dzhanabekova R.Kh., Seliverstova E.V., Zhumabekov A.Zh., Ibrayev N.Kh. // Rus. J. Phys. Chem. A. 2019. V. 93. N 2. P. 338. doi 10.1134/S0036024419020092

[35] Dzhanabekova R.Kh., Seliverstova E.V., Ibrayev N.Kh. // Rus. J. Phys. Chem. A. 2017. V. 91. P. 1761. doi $10.1134 / \mathrm{S} 003602441709028 \mathrm{X}$

[36] Kokil A., Yang K., Kumar J. // J. Polym. Sci. Part B. 2012. V. 50. P. 1130. doi 10.1002/polb.23103

[37] Li Sh., Tao D., Zhang Ya., Li Y., Yin W., Chen Q., Liu Z. // Nanophot. 2019. V. 8. N 5. P. 899. doi 10.1515/nanoph-20190060

[38] De Sanctis A., Mehew J.D., Craciun M.F., Russo S. // Materials. 2018. V. 11. N 9. P. 1762. doi 10.3390/ma11091762

[39] Patil V., Capone A., Strauf S., Yang E.H. // Sci. Rep. 2013. V. 3. P. 2791. doi $10.1038 /$ srep02791

[40] He T., Zhao Y., Zhang X., Lin W., Fu K., Sun C., Shi F., Ding X., Yu G., Zhang K., Lu Sh., Zhang X., Zhang B. // Nanophotonics. 2018. V. 7. N 9. P. 1557. doi 10.1515/nanoph2018-0061

[41] Kong X., Liu C., Dong W., Zhang X., Tao Ch., Shen L., Zhou L., Fei Y., Ruan Sh. // Appl. Phys. Lett. 2009. V. 94. P. 123502. doi $10.1063 / 1.3103288$

[42] Ahmad H., Tajdidzadeh M., Thambiratnam K., Yasin M. // Laser Phys. 2018. V. 28. P. 066204. doi 10.1088/15556611/aab451

[43] Thomas S., Sakho E.H.M., Kalarikkal N., Oluwafemi O.S., $W u$ J. Nanomaterials for Solar Cell Applications. Amsterdam: Elsevier, 2019. $534 \mathrm{p}$.

[44] Novoselov K.S., Geim A.K., Morozov S.V., Jiang D., Zhang Y., Dubonos S.V., Grigorieva I.V., Firsov A.A. // Science. 2004. V. 306. N 56996. P. 666. doi 10.1126/science.1102896.

[45] Maiti R., Sinha T. K., Mukherjee S., Adhikari B., Ray S.K. // Plasmonics. 2016. V. 11. P. 1297. doi 10.1007/s11468-0150175-0

[46] Liu Y., Cheng R., Liao L., Zhou H., Bai J., Liu G., Liu L., Huang Y., Duan X. // Nat. Commun. 2011. V. 2. P. 579. doi $10.1038 /$ ncomms 1589

[47] Echtermeyer T.J., Britnell L., Jasnos P.K., Lombardo A., Gorbachev R.V., Grigorenko A.N., Geim A.K., Ferrari A.C., Novoselov K.S. // Nat. Commun. 2011. V. 2. P. 458. doi $10.1038 /$ ncomms 1464 